\title{
sciendo
}

\section{The history of economics and the Constructal Theory}

\author{
Radu ISAIC \\ Bucharest University of Economic Studies, Bucharest, Romania \\ isaicradu@yahoo.com \\ Cristian PĂUN \\ Bucharest University of Economic Studies, Bucharest, Romania \\ cristian.paun@rei.ase.ro
}

\begin{abstract}
The signs of a new crisis appear. Some countries are moving towards real assets (gold, oil); the real estate market froze, as did the credit market; sales are on a global decline. The world has become accustomed to economic cycles and crises. At the theoretical level, efforts are aimed at finding practical methods of prevention and especially of combating the negative effects on economic cycles. The market of theories regarding the internal causes and mechanisms of economic cycles has stabilized. The theoretical barricades were formed and strengthened and now the projectiles are thrown from one side to another. Demand theories and supply theories give partial explanations, but with claims of total theories. Is it possible to see something new at the theoretical level about the causes and mechanisms of economic cycles? We think so. We believe that the Constructal Theory of Professor Adrian Bejan has an explanatory power over the present theories about the causes of the economic cycles. The perspective offered by this theory is new and offers explanations for aspects that are difficult to understand in other theories. Through this article we want a rewriting of economic history, from the perspective and through the lens of this theory. We believe that we offer a broader and deeper explanation of the causes of economic cycles using a theoretical tool borrowed from other sciences, but with great explanatory powers in the social sciences. We will rewrite the economic history by discussing: S-evolution of economic stages; overlapping economic flow systems; systems evolving from one another; increased resource consumption and increased resource efficiency. The results of this study indicate that abusive interventions in the economic system create problems. The economic system, which is essentially a flow system, like any other system, must be allowed to evolve freely.
\end{abstract}

Keywords: flow systems, economic cycles, money, central banks, braking and acceleration systems.

\section{Introduction}

By this article the authors will try to take a new look and to interpret in a different way the history and especially the economic history from the perspective of the Constructal Theory of Professor Adrian Bejan. The article follows two articles written by the authors in which they presented the basis of thinking of the mentioned theory. In this article we will try to apply the theory concretely.

Constructal Theory speaks in terms of movement as the essence of life, movement that is organized in flow systems. Death means stagnation, cessation of movement. To have movement there must be a motor and a brake in the system; one without the other is not possible.

There is no displacement without the conceptual understanding of the separation between the thing being transported and the material that transports. The movement exists only in a relative space: in which things move relative to each other.

To understand C.T. we must understand that nothing jumps from one place to another without something to transport it. Even the image of a thing must be worn. The man must transport himself to food sources; sources that are moving compared to man or to each other. The 
man must carry the objects of his pleasures for possible late consumption. Man is limited around him.

Things get a bit complicated when people start carrying images. Image is the true definition of information. The image is a map (a sketch) of what is really (in reality; in its existing form) somewhere, at some distance of travel.

What images do people move? The image of welfare, that is, the image of the existence of

PICBE | 869

goods (resources) that satisfy the needs of people. How are these images carried? By what is called, generically, money. Money is the bearer of images, information, ideas (the idea understood as the mental action on some goods).

The authors of this article presented C.T. in two previous articles so that from space economy in this article we will no longer present the basics of C.T.

\section{Literature review}

\section{Economy in the beginning period}

The first testimonies about human existence place him in a position of hunter gatherer. In this capacity, in order to survive, he had to constantly move in order to find the ones needed for living. The human species has thus moved across the entire surface of the globe.

The first freight was just what people could carry; no burden animals existed. Probably slight exchanges were made directly in nature, based on a primitive negotiation. The move was according to T.C. from a point on the whole surface. As long as there were no barriers to displacement and population growth, everything was fine.

The startup economy was based on local random shifts, but we believe, everyone was trying to avoid wanting to remain self-sufficient. Avoidance was based on fear of possible violence. The exploitation of resources was superficial due to their abundance.

In this beginning period, the first information transport was also carried out in the form of stories (about facts, happenings, places). Being in a permanent movement probably no common measurement standards or even common values were made.

Continuing the argumentation to understand better, we will keep in mind: a large flat surface, with unevenly distributed resources (the most important aspect) and on which people are spread evenly (contrary to the way resources are spread).

Territorial beings, people need a certain surface in order to survive. In the beginning the area must be large because the capital goods are primitive. Step by step the population density increases.

Due to the resources distributed randomly and unevenly, the following phenomena occur.

In areas with scarce resources the density is low per unit area (large areas are needed to support a human life), demographic control is used through the killings of infants and capital goods have a slow evolution.

In resource areas, their operation takes place in discrete steps. But different types of resources are in different areas. The human mind (the first and most important resource) is uniformly distributed, but is bound to change unevenly due to the resources it is required to work with. Different resources plus the human mind, lead to different capital goods.

Next, the evolution is about the concrete way in which: the place where the resources are located; their concentration on the surface unit; the distance between the resource location points; their dispersion; the existence of other types of resources between the centers of location of the large concentrations of resources.

\section{Economy, thinking from big to small}


Large resource concentration centers acted as large mass concentrations in relativity theory. They drew resources from the surrounding areas; these were attracted, modified and scattered in the neighboring areas.

The innovations are punctual, they refer to local resources. In order to be useful, innovations must be attracted by the large agglomerations of capital goods. That is: to be transformed and especially integrated into the whole of capital goods (like a puzzle game).

PICBE | 870

Armed with the above, we understand the history of humanity: with large cultural centers (resource concentrations); determinant is the way of spreading and integrating the innovations in the previous systems; evolution is in steps in some areas and the apparent refusal to evolve in others.

America has few resources (resources that can be exploited in the first instance) and with their north-south distribution, it has led to a blockage. That is, the innovations are tailored to local resources and could not be spread.

Europe has seemingly unlimited resources and benefits from the accumulation of all the innovations due to the east-west form of the Eurasian continent.

All the great civilizations had an evolution in the form of S. It starts with a slow growth, and from a certain turning point where a critical mass is reached (accumulation of positive effects of innovations), the evolution accelerates, at an increasing rate. The next step is: the reach of a new critical mass (accumulation of negative phenomena), where a new critical point is reached, from which the evolution becomes again slow; the growth is at a decreasing rate. There are no hits of a wall (cataclysms), and from a certain point another type of evolution is superposed over the initial one. The old system will still work, but the main purpose of facilitating the flow will be taken over by the new, more efficient system.

The most important political innovation that facilitated the flow was the EMPIRE. The empire has determined: a unitary language that helps to transmit messages in space and time; unity of laws; unit of measurement standards; unit of monetary systems; unity of beliefs and moral values. All these uniformities in human activities facilitated the flow.

Perhaps the most important aspect of humanity is exchange. Exchange of ideas, values, capital goods, consumer goods. The exchange means: displacement (of a good, resource) from places with high concentration to places with low concentration. There is a unique motivation for moving and exchanging: differences in resource concentration.

\section{Economy from small to large}

To simplify the presentation, we assume the following: the land surface has uniformly distributed resources, there is no difference either in climate, relief or different kinds of resources between areas, and people are spread widely. equal on the earth's surface like the nodes of a perfectly symmetrical network.

We could think that we would have an identical system with itself for an indefinite period of time. Equal resources involving equal consumer goods and identical capital goods. The only way to have more would be through the robberies of the neighbors.

A single factor of production cannot be equal (no matter how hard we strive): the human mind. Birth differences in environmental exploitation capabilities are different between people. And the abilities born to some can be transmitted culturally to those around the original individual. Cultural heritage is formed. 
Differences in the way of exploiting the resources will appear randomly on this presumed surface. Some more creative and especially visionary individuals will create capital goods of different types and models.

To illustrate: suppose that in some place an individual innovates, with the resources he has available and forms a new capital good and a new consumer good. From here on, we will enter the field of classical economics. That is, people will start to change between them. When the phenomenon of diffusion reaches a critical mass, the first channels of movement and transport are formed.

The human mind has different abilities, so some individuals are inclined to take risks on freight transport and efficient trading. On a uniform surface, inevitable concentrations of capital goods and modes of exploitation occur. People inevitably tend to approach each other to facilitate exchange. The exact place where those mentioned concentrations appear is impossible to anticipate. The human capacities to establish human relations and to leave their inheritance are different.

When forming the centers (cities), they tend to attract the surrounding individuals. In cities, the flow is much accentuated due to the proximity and diversity of the hierarchically inferior systems. The attraction rate decreases with the distance from the center (cities). The tendency is to cover the entire surface. So the centers have certain dimensions. From many small, to a few large, with a certain rate between size / number. Flow systems attempt to cover the entire surface so that all resources are exploited as efficiently as possible. Between the centers flow channels are formed for the exchange of goods / information.

In continuation of the above reasoning. First small centers are formed on a small surface. These small centers have little capital goods because the related area and resources are scarce. Suppose we double the surface, so larger centers appear. They process more resources; processes the initial resources in a secondary and tertiary way; processes resources that in the first centers could not be exploited economically, etc. The type of argument can continue in the same way. The main idea is to spread the centers in such a way that they can be exploited and covered the whole area and exploited in the most efficient and productive way. The distribution curve of the centers is thus formed: from many small to few large ones.

The different ways of thinking, inside the centers, will lead to the emergence of specializations. Some goods appear in one area and are missing in another. So, like the classical economic theory and the classical laws of physics: things move from high concentrations to low concentrations.

Means of transport refer to the frictional forces in physics. Their evolution depends on innovations. The robberies are in physical terms the loss of energy to the environment. The system seeks to limit losses so that centers spread their jurisdictions to the limit of meeting with other centers.

The means of transport are made by each center according to its size and are formed to the extent of its jurisdiction. Thus, flow paths (roads, canals) appear, from many small to few large ones, with a certain rate of decline.

The limiting factors in establishing a perfect mathematical model of the spread, in the real world of centers and paths are: geography; random distribution of resources; climate; random spread of human skills. 


\section{The Role of Money in Constructal Theory}

The role of money in the life of society fits perfectly within C.T.. Money is an innovation to facilitate the flow within the flow system formed by human society.

The materialization of the concept of money has been flexible throughout the evolution of human society. Different categories of materials have been used as means of exchange in various places. Diversity probably depends on local resources and local beliefs / values. When different areas with the related centers are definitely connected, there is a competition between the various categories of money. Which material wins this competition is less important. It happened to be gold, probably a human affinity for gold, an affinity whose internal mechanisms do not belong to the economic field.

Money, like other innovations mentioned in these articles, was an inevitable innovation, due to the fluidization of the flow in the system of human society. There are numerous advantages of using the money presented in the literature, so they will not be mentioned here.

The most important aspect of the money is that they formed flow channels, parallel to the flow channels of the goods. The two types of channels are interconnected in the sense that one determines the other. That is, the movement in the money channel determines the type of movement in the goods channel.

We must answer the questions specified in the introduction:

1 Through financial channels, money is transported (in the form of a commodity of exchange value), in relation to, or in comparison with, what consumer goods or capital goods.

2 Through the movement of the money entity, information related to the existence of certain goods is carried.

3 The amount of money, which is held / carried, is given by the power of storing the information on the unit of money (the evolution was in the direction of making this transport more efficient).

4 The place of movement. As money was initially a commodity, the movement of money accompanied the movements of other goods. Then with money abstraction, the channels of money flow separated; reaching a system of flow totally separate from that of the goods; system totally dependent on technological innovations.

5 The material of which the channel is composed. It started in the form of roads on which the goods were transported and ended in computer innovations that turned money into computer codes.

6 Material of the flow. Initially it was a commodity; it came in the form of symbols of economic power, from a certain area.

7 The forces opposing the movement. It started as forces opposing the movement of any commodity (movement mechanisms, road safety, legislation of various areas regarding the movement of goods), until the Central Banks legislation and regulations on the monetary mass as a whole.

8 The frictional forces and resistance encountered during the flow. It is given (at this moment) by the regulations and norms of the Central Bank that controls the monetary mass in a certain area. Initially it was given by the safety and security of freight transport, with great value.

9 Flow function. It transmits information about the scarcity of resources and the intensity of needs. It engages the movement of goods, with a concrete existence.

10 The flow stops are given by the rules of the Central Bank and especially by the interest rate. 
We will go through the period (we consider it uninteresting) to find a value - a commodity that will take over the role of money. From the moment that gold took over this role, its evolution becomes somewhat predictable. His movement is concentrated in places with high concentrations of human activities. Gold accompanies ordinary freight, being traded on ordinary freight. Gold (and other goods) move from high-concentration places to low-concentration places. There is a concentration of gold in certain places (temples - banks) as security measures. In every administrative area there is the legislation necessary to regulate the commodity gold.

Throughout its existence, gold - money, must keep in touch with commodities. Money must be the image of goods. There must be similarity and correspondence between money and goods. Due to precisely the qualities for which they were selected, gold - money has a more intense circulation than the goods, it moves and deposits more easily. Essentially they get easy from one place to another or from one owner to another, without the corresponding real goods being moved (moving the goods depends on the existing technology)

Parallel flow channels of money form centers parallel to those of other goods and not necessarily in the same places. A parallel world is formed; world with its own rules and regulations. In a perfect world there should be a permanent correlation between the two channels of flow. But the money gets their own turbidity.

The big problem: access to the goods flow channels can only be done indirectly through the money flow channels.

Important aspects in short. The price of a product, does not represent the energy stored in that product, in fact it is a flow of energy that starts from one point and reaches another. Saving is saved power of movement. If one place consumes more energy than it needs for that place, then the energy (which is in addition) moves in the form of economy to another place, where it is needed. If in a place energy is created over needs then it is necessary to store it and use it later (possibly at another time or place).

Money transfers movement power from those who hold it in excess, to those who use it now or in the future.

The written norms and laws, especially the unwritten ones, form flow channels and lead to: the longitudinal flow to be easy (along the channel); cross-sectional losses (outward) to be reduced. The business world (ie the exchange of matter / information) and especially of its rules (I repeat the written and unwritten ones) generate a system of channels whose sole purpose is to facilitate the flow of matter / information.

The next logical step is: the problem of rules. If the rules came into being spontaneously (from the collaboration of generations and in which each one contributed without being able to identify a particular person), then the system as a whole is most likely to be efficient, stable and facilitates flow. A culture as a whole, of individuals and generations (generation that have an invisible connection between them), has its own intelligence. This intelligence is the source of some rules, which aim towards perfection in the accessibility of the whole territory; regarding the flow of goods and information (flow that is constantly evolving and adapting to local conditions).

The rules formed rationally, by a person or a group of persons, are subject to the inherent mistakes concerning: the limited intellectual capacities of a person; limited knowledge from a historical stage; the preconceived ideas of a culture at a historical stage.

These are the differences between the monetary system based on gold and the monetary system based on the regulations of the Central Bank. As C.T. argues, the destruction of local systems, which have proven effective over time and the construction of new ones that are effective elsewhere, leads to devastating effects. 
The money flow system is one with a certain hierarchy and architecture. It evolves (if it is left free) over time in steps impossible to anticipate, but it can only give the tendency: to increase the flow.

These rules apply from the exchange of money (the loan) between friends and acquaintances, to the flow of money with the state (in the form of taxes and taxes), to the flow between individuals and companies, to the flow between companies.

PICBE | 874

\section{Methodology}

Professor Andrei Bejan, through his work in the field of thermodynamic engineering and physics, has surprised, through an intuitive vision, how the flow systems exist and manifest themselves. We can speak of an Aristotelian-style surprise of the essences of the real world by a mind in constant search of causal connections and configurations.

Until C.T. it was believed that the flows in various systems are random. C.T. proves that absolutely in all flow systems there are mathematical correspondences between the various parameters. Mathematical laws (on which flow systems are based) are accurate and can define certain trends.

Generally, during the evolution of a theory, first the theoretical bases are put in the form of hypotheses, then empirical and mathematical evidence is presented. Usually, the differences in one stage and the other, are of a generation even two. R.T. it has the chance that, in the same generation, the theoretical bases (hypotheses) will be laid and empirical evidence will be brought

\section{Results and discussions}

How do crises appear in a vision of human society like a flow system (generally tree-shaped)? We believe that there are three factors that lead to the crisis: innovation, administrative bureaucracy and central banks.

All three causes affect the flow of goods and services / information differently. All block existing flow from certain areas and / or activate the flow in low flow areas or areas without flow. In order to understand the future reasoning, we must accept that the flow system is permanently adapted to the existing conditions (for the most efficient flow). The conditions are in permanent change, so the flow adapts and evolves in steps to change, but inevitably it is one step behind the changes. Sudden or unnatural changes create crises in the flow system, which takes time to adapt to new conditions. During the crises there will be individual sacrifices, but what matters is the system as a whole. The system naturally seeks to reduce losses and forces that oppose the flow.

1 Innovation. It can be in the field of resource exploitation or in the field of transport. Innovations bring new resources to the market or more efficiently exploit the old ones. Innovations in the field of transport: move more goods with less energy consumption. Regardless of innovation, there is an increased inflow of goods into the flow system. Old channels are forced to the max. It is necessary to create new channels and especially to create channels in areas where there were not or were few. The innovations extend the center and widen the periphery of a culture. Naturally, the new flow of matter seeks to occupy as much space as possible and expands into white areas. Previously heavily vascularized areas may stagnate due to pre-existing channels that can no longer be changed; instead the flow starts towards new areas. The innovations generate new flow systems superimposed on the old ones, which they do not replace. This is exactly what happens in the existence of successive cultures. 
2 Administrative bureaucracy. Bureaucracy acts through laws that normally should make the flow accessible to different areas, in particular they block the flow. Raising barriers to freight transport blocks the flow. Bureaucracy creates insurmountable barriers: customs (import and export), monetary, cultural, linguistic, measuring units; generates bottlenecks in the flow path. These lead to hypervascularity of some areas and undevelopment of others. In the centralized state, the center is artificially developed and the other areas are poor (areas for which no channels are built). This is exactly what is happening in the European Union.

3 Central banks. To understand the role of C.B. we must start with the beginning of money - commodity - gold. Once gold is established as money, it has created its own flow system, due in particular to the implicit value contained in it. Starting with temples, various physical persons and ending with modern banks, all these entities functioned as nodes of a network of a flow system. The flow channels were formed from the receipts proving the existence of a certain quantity of gold deposited in a certain place. The individuals through whose hands the receipts passed were smaller, hierarchically inferior knots.

By itself the gold flow system is of no importance, but connected to the commodity system, becomes of paramount importance. Gold, as a proof of the existence of a specific consumer good / capital, will determine its existence. Gold provides information about what is happening in the world of goods.

From the moment the gold depositors issued more receipts than the deposited gold, problems arose. The issuance of additional receipts was achieved primarily by violating the property rights of the depositors (an aspect of first importance insufficiently emphasized). Thus, in the money flow system, the flow was artificially increased, and the flow system for goods remained constant. Thus, the correspondence between the two interdependent flow systems (money and goods) was no longer maintained. The different travel speed and the formation of distorted (corrupt) images between money and goods led to crises.

Gold as a concrete existence represented the braking system of the money flow system (a system to operate needs two systems: engine and braking). Because the gold commodity could not be increased randomly and because there were automatic adjustments between banks regarding the amount of receipts issued on the gold deposits, the difference between goods and receipts could not be too great. Thus, the crises were local and small.

Over the old system of flow with parallel and equivalent channels (gold - goods), a new system of flow was deliberately and artificially superimposed: the Central Bank's rules. The genesis of the new system we consider: 1914 (the establishment of the FED) and has an evolution in the form of S (slowly - rapidly - slowly).

To begin with, there were two concurrent braking systems: gold and C.B. regulations. Like two antivirus systems in a computer they blocked the economic system. The result is the succession of crises in the interwar period that ceased only when one of the braking systems was practically canceled by the Treaty of Breton Woods (1944). The period of slow growth we consider it 1914 - 1944. With 1944 the accelerated growth begins. The brakes of the world money flow system (and implicitly of the goods) are represented by the C.B. regulations

Starting with 1990 we consider that it has entered on the second phase of slow growth and now we are at the end of the line that expresses the evolution of this system. Why ? Because the regulatory and understanding capacities are inherently limited. The rules of the Central Bank were at the same time the engine and brake of the economic flow system. Sometimes alternatively, sometimes concurrently. 
C.B. are now required to assume the role and functions of gold (stabilizer and brake). In fact, C.B. diluted the braking system (of the monetary flow system) and strengthened the acceleration by permanently increasing the money supply.

A new flow system can be seen and that will overlap the old one. It's about cryptocurrencies. They will accumulate confidence as old coins lose theirs, due to poor management. The systems overlap each other, without being replaced. The paper money system did not lead to gold being thrown out of banks or loans between states without a real guarantee. So probably in the future we will have gold (or other real assets), fiduciary currency (guaranteed by a state through the economy as a whole) and cryptocurrencies.

A crisis is a necessary re-alignment of the correspondences between the two parallel flow systems (money and goods). Each system offers and receives information about the status of the other. The solution is to leave the two systems a high degree of freedom so that they can achieve harmony between them; to find the natural path of minimum resistances.

As Marx predicted, the monetary system became more abstract with the passage of time, lost contact with the concrete reality and created crisis after crisis. The future is an even greater abstraction of money. Money from the merchandise has gone to paper printed with symbols of the economic power of a state, then to electrical impulses managed by a hand of people and will pass to electrical impulses managed by nobody. The problem is the disappearance of the flow braking system, its dilution until not identified. A car with only the accelerator pedal does not have a long life or to go safely it runs at an extremely low speed.

\section{Conclusion}

The solution for poverty eradication is not the artificial modification of the flow channels but the connection of the isolated areas to the high flow systems; the connection is made from step to step, ie from low to high with a certain rate. The solution is to develop local flow systems (not destroy them) and connect them to larger flow systems.

In the action of extending the monetary mass (realized by C.B.), the phenomenon of income suction appears from those who save (the classes with small and medium incomes) to those with high incomes (investors).

The social system must be left as free as possible, without bureaucratic interventions. Thus, he will find the path to a flow as easy and efficient. C.T. draws near as conclusions to the Austrian School of Law and Economics.

By forcing the flows only in a certain direction (administratively an economic sector is preferred), the artificially vascularized area over potential will withdraw resources from other areas.

In order to reduce the size of the crises and their effects C.B. must take over the function of gold and tightly regulate the money circulation system. Of course with the negative changes mentioned above. The abnormal evolution against nature of the economic system that will inevitably lead to another type of crisis (impossible to predict) is the price of giving up gold.

\section{References}

Bejan, A., \& Zane, P. (2012). Design in nature. How constructal law governs evolution in biology, physics, technology, and social organization. New York: Anchor Books.

Bejan, A. (2016). The Physics of life. New York City St.: Martins Press.

Bejan, A, Errera MR. Wealth ineguality: The physics basis. Journal of Applied Physics 121, 
124903 (2017); doi: 10.1063/1.4977962. http://dx.doi.org/10.1063/1.4977962. http://aip.scitation.org/toc/jap/121/12. American Institute of Physics

Bejan, A, Gunes U, Errera MR, Sahin B. Social organization: The thermodynamics basis. Int J energy Res. 2018; 42: 3770-3779. http://doi.org/10.1002/er.4093.

Bejan, A. (2020). Fredom and Evolution. Hierarchy in Nature, Society and Science. Gewerbestrasse: Springer Nature Switzerland.

Bejan, A, Errera MR, Gunes U. Energy theory of periodic economic growth. Int $J$ Energy Res. 2020; 1 - 12. https://doi.org/10.1002/er.5267

Friedman, M. (1982). Capitalism and Liberty. Chicago: The University of Chicago.

Hayek, F. (1998). Prices anf Production. London: Routledge.

Keynes, M. (2007). The General Theory of Employment, Interest and Money. London: The Royal Economic Society.

Minsky, H. (2008). Stabilizing An Unstable Economy. New York: McGraw-Hill Education. Mises, von L. (1966). Human Action. Chicago: Henry Regnery.

Soto, H. (2006). Money, Bank Credit, and Economic Cycles. Alabama: Ludwig Von Mises Institute 